\title{
The Equivalence of the Minimal Dominant Set and the Myopic Stable Set for Coalition Function Form Games
}

Citation for published version (APA):

Herings, P. J-J., \& Kóczy, L. Á. (2020). The Equivalence of the Minimal Dominant Set and the Myopic Stable Set for Coalition Function Form Games. Maastricht University, Graduate School of Business and Economics. GSBE Research Memoranda No. 017 https://doi.org/10.26481/umagsb.2020017

Document status and date:

Published: 09/06/2020

DOI:

10.26481/umagsb.2020017

Document Version:

Publisher's PDF, also known as Version of record

\section{Please check the document version of this publication:}

- A submitted manuscript is the version of the article upon submission and before peer-review. There can be important differences between the submitted version and the official published version of record.

People interested in the research are advised to contact the author for the final version of the publication, or visit the DOI to the publisher's website.

- The final author version and the galley proof are versions of the publication after peer review.

- The final published version features the final layout of the paper including the volume, issue and page numbers.

Link to publication

\footnotetext{
General rights rights.

- You may freely distribute the URL identifying the publication in the public portal. please follow below link for the End User Agreement:

www.umlib.nl/taverne-license

Take down policy

If you believe that this document breaches copyright please contact us at:

repository@maastrichtuniversity.nl

providing details and we will investigate your claim.
}

Copyright and moral rights for the publications made accessible in the public portal are retained by the authors and/or other copyright owners and it is a condition of accessing publications that users recognise and abide by the legal requirements associated with these

- Users may download and print one copy of any publication from the public portal for the purpose of private study or research.

- You may not further distribute the material or use it for any profit-making activity or commercial gain

If the publication is distributed under the terms of Article $25 \mathrm{fa}$ of the Dutch Copyright Act, indicated by the "Taverne" license above, 
P. Jean-Jacques Herings,

László Á. Kóczy

The Equivalence of the Minimal Dominant Set and the Myopic Stable Set for Coalition Function Form Games

RM/20/017

ISSN: $2666-8807$

\section{GSBE}

Maastricht University School of Business and Economics

Graduate School of Business and Economics

\section{P.O Box 616}

NL- 6200 MD Maastricht

The Netherlands 


\title{
The Equivalence of the Minimal Dominant Set and the Myopic Stable Set for Coalition Function Form Games*
}

\author{
P. Jean-Jacques Herings ${ }^{\dagger} \quad$ László Á. Kóczy
}

May 29, 2020

\begin{abstract}
In cooperative games, the coalition structure core is, despite its potential emptiness, one of the most popular solutions. While it is a fundamentally static concept, the consideration of a sequential extension of the underlying dominance correspondence gave rise to a selection of non-empty generalizations. Among these, the payoff-equivalence minimal dominant set and the myopic stable set are defined by a similar set of conditions. We identify some problems with the payoff-equivalence minimal dominant set and propose an appropriate reformulation called the minimal dominant set. We show that replacing asymptotic external stability by sequential weak dominance leaves the myopic stable set unaffected. The myopic stable set is therefore equivalent to the minimal dominant set.
\end{abstract}

KEYWORDS: coalition structure core; sequential dominance.

JEL CODES: C71.

${ }^{*}$ Kóczy thanks the support of the National Research, Development and Innovations Office (NKFIH) under grant number K-128573. This research was supported by the Higher Education Institutional Excellence Program of the Ministry for Innovation and Technology in the framework of the "Financial and Public Services" research project (reference number: NKFIH-1163-10/2019) at Corvinus University of Budapest.

${ }^{\dagger}$ P.J.J. Herings, Department of Economics, Maastricht University, P.O. Box 616, 6200 MD, Maastricht, The Netherlands. P.Herings@maastrichtuniversity.nl

${ }^{\ddagger}$ L.Á. Kóczy, Centre for Economic and Regional Studies, Tóth Kámán u. 4., 1097 Budapest, Hungary and Department of Finance, Faculty of Economics and Social Sciences, Budapest University of Technology and Economics, Magyar tudósok körútja 2, 1117 Budapest, Hungary. koczy@krtk.mta.hu 


\section{Introduction}

The core is one of the most popular solution concepts in cooperative game theory. It introduces a coalitional notion of stability not unlike the Nash equilibrium: once such a proposal is made, it is not abandoned. The similarities go further: neither of these concepts tell us how such agreements are reached. Suppose the game starts with an arbitrary proposal: how to move from this proposal to an acceptable, equilibrium allocation?

To our knowledge Stearns (1968) was the first to look at this problem by considering a recontracting process where myopic deviations by coalitions are considered as improvements. Green (1974), Neuefeind (1974), and Wu (1977) present results that such improvements almost surely or approximately reach the core. The results of Arnold and Schwalbe (2002) extend even to NTU-games. More recently, Roth and Vande Vate (1990), Diamantoudi, Miyagawa, and Xue (2004), Klaus and Klein (2007) and Chen, Fujishige, and Yang (2016) show for matching markets and Sengupta and Sengupta (1994, 1996), Kóczy and Lauwers (2004), and Kóczy (2006) show for coalitional games that the core can actually be reached. The latter results prove the existence of an upper bound on the number of steps needed. This bound has since been drastically lowered (Yang, 2010, 2011; Béal et al., 2012, 2013a,b).

It is natural to ask where do these coalitional improvements lead in case the core is (possibly) empty. Sengupta and Sengupta (1994) define the set of viable proposals, Kóczy and Lauwers (2007) the payoff-equivalence minimal dominant set ${ }^{1}$ and most recently Demuynck, Herings, Saulle, and Seel (2019) the myopic stable set. All these concepts rely on notions of sequential dominance and belong to the family of stochastic solutions (Packel, 1981) with appropriate restrictions on the permitted transitions.

We identify a problem in the definition of the payoff-equivalence minimal dominant set; under the current definition Theorem 4 of Kóczy and Lauwers (2007) does not hold. The problem is caused by the incorporation of payoff equivalent outcomes in the dominance correspondence. We argue that payoff equivalence should be dropped, which leads to the notion of the minimal dominant set. We go on to show that the minimal dominant set and the myopic stable set are equivalent, where for the latter concept it does not matter whether strict or weak dominance is used in the definition. It then follows from Yang (2020) that both concepts are included in the set of viable proposals.

Accordingly, the structure of the paper is as follows. First, we introduce the basic terminology and notation together with both the payoff equivalent minimal dominant set and the minimal dominant set. Then we introduce the myopic stable set and its variant based on weak dominance called the weak dominance myopic stable set. Finally, we present

\footnotetext{
${ }^{1}$ Kóczy and Lauwers (2007) use the terminology minimal dominant set, but following the later literature we reserve this term for a different concept.
} 
our main result showing the equivalence of the minimal dominant set, the myopic stable set, and the weak dominance myopic stable set.

\section{The Payoff-equivalence Minimal Dominant Set}

A characteristic function form game is defined by a pair $(N, v)$, where $N$ is a set of players with cardinality $n$ and a characteristic function $v: 2^{N} \rightarrow \mathbb{R}$ that assigns to each coalition $C \subseteq N$ a number $v(C) \in \mathbb{R}$, called the worth of $C$, with the usual convention that $v(\emptyset)=0$. The collection of non-empty coalitions is denoted by $\mathcal{N}$. A coalition structure is a partition $\mathcal{P}=\left\{C^{1}, \ldots, C^{m}\right\}$ of $N$. It describes how the grand coalition is divided into non-overlapping subcoalitions. The collection of all coalition structures, that is, the collection of partitions of $N$, is denoted by $\Pi$. For a coalition $C \in \mathcal{N}$, let $\langle C\rangle$ denote the partition into singleton coalitions. Formally, we have that $\langle C\rangle=\{\{i\} \mid i \in C\}$.

For a coalition structure $\mathcal{P} \in \Pi$ and a coalition $D \in \mathcal{N}$, the partners' $\operatorname{set} P(D, \mathcal{P})$ of $D$ in $\mathcal{P}$ is the union of those coalitions in $\mathcal{P}$ that have a non-empty intersection with $D$, the residual players $R(D, \mathcal{P})$ are the players in the partners' set outside $D$ and the outsiders $O(D, \mathcal{P})$ are all the players outside the partners' set, so more formally we have

$$
\begin{aligned}
& P(D, \mathcal{P})=\underset{C \in \mathcal{P}, C \cap D \neq \emptyset}{\bigcup} C, \\
& R(D, \mathcal{P})=P(D, \mathcal{P}) \backslash D, \\
& O(D, \mathcal{P})=N \backslash P(D, \mathcal{P}) .
\end{aligned}
$$

The collection of coalitions to which the outsiders belong is given by

$$
\mathcal{O}(D, \mathcal{P})=\{O \in \mathcal{P} \mid O \cap D=\emptyset\}
$$

For coalition function form games, we define the set of outcomes $X$ as the set of coalition structures $\Pi$ together with all individually rational payoff vectors that can be obtained by allocating the worths among the members of the respective coalitions:

$$
X=\left\{(\mathcal{P}, u) \in \Pi \times \mathbb{R}^{N} \mid \forall i \in N, u_{i} \geq v(\{i\}) \text { and, } \forall C \in \mathcal{P}, \quad \sum_{i \in C} u_{i}=v(C)\right\} .
$$

Given an outcome $x \in X$, we denote by $\mathcal{P}(x)$ the projection to its first component, that is, the coalition structure, and by $u(x)$ the projection to its second component, that is, the payoff vector. We can thus write $x=(\mathcal{P}(x), u(x))$. The set $X$ is non-empty since it always contains the outcome $x$ where $N$ is partitioned into singletons, so $\mathcal{P}(x)=\langle N\rangle$, and each player $i \in N$ receives the payoff $v(\{i\})$.

Kóczy and Lauwers (2007) define the notion of outsider-independent dominance, to which we will refer as weak dominance in this paper. 
Definition 2.1. An outcome $y \in X$ weakly dominates an outcome $x \in X$ by deviating coalition $D$ if

$$
\text { - } \mathcal{P}(y)=\{D\} \cup\langle R(D, \mathcal{P}(x))\rangle \cup \mathcal{O}(D, \mathcal{P}(x)),
$$

and

- for every $i \in D, u_{i}(y) \geq u_{i}(x)$ and there exists $i \in D$ such that $u_{i}(y)>u_{i}(x)$,

- for every $i \in O(D, \mathcal{P}(x)), u_{i}(y)=u_{i}(x)$.

After a coalition $D$ deviates, the residual players fall apart into singletons, whereas the outsiders stay in their original coalitions. Utilities of outsiders are not affected, whereas the utilities of the deviating players all weakly improve and at least one deviating player strictly improves. Since $y \in X$, it follows from Definition 2.1 that $\sum_{i \in D} u_{i}(y)=v(D)$ and, for every $i \in R(D, \mathcal{P}(x)), u_{i}(y)=v(\{i\})$. The treatment of residual players corresponds to the $\gamma$-model of Hart and Kurz (1983). The property that outsiders are not affected by a coalitional deviation is also referred to as coalitional sovereignty in Konishi and Ray (2003), Ray and Vohra (2014, 2015), and Herings, Mauleon, and Vannetelbosch (2017).

One of the most prominent set-valued solution concepts for coalition function form games is the coalition structure core as defined in Aumann and Drèze (1974).

Definition 2.2. The coalition structure core (CSC) of $(N, v)$ is the set of outcomes $x \in X$ such that, for every coalition $C \in \mathcal{N}$,

$$
\sum_{i \in C} u_{i}(x) \geq v(C) .
$$

In words, the coalition structure core gives to the members of each coalition at least the payoff they can obtain by forming that coalition. It is easy to provide examples where the coalition structure core is empty.

Let some outcome $x \in X$ be given. The set of all outcomes that weakly dominate $x$ together with outcome $x$ itself is denoted by $g(x)$, so

$$
g(x)=\{x\} \cup\{y \in X \mid \text { there is } D \in \mathcal{N} \text { such that } y \text { weakly dominates } x \text { by } D\} .
$$

We define the two-fold composition of $g$ by $g^{2}(x)=\{z \in X \mid \exists y \in g(x)$ such that $z \in$ $g(y)\}$. By induction, we define the $k$-fold iteration $g^{k}(x)$ as the subset of $X$ that contains all outcomes obtained by a composition of dominance correspondences of length $k \in \mathbb{N}$, that is, $g^{k}(x)=\left\{z \in X \mid \exists y \in g^{k-1}(x)\right.$ such that $\left.z \in g(y)\right\}$. Observe that for every $k, \ell \in \mathbb{N}$ if $k \leq \ell$, then $g^{k}(x) \subseteq g^{\ell}(x)$. We define the set of all outcomes that can be reached from $x$ by a finite number of dominations by $g^{\mathbb{N}}(x)$, so

$$
g^{\mathbb{N}}(x)=\bigcup_{k \in \mathbb{N}} g^{k}(x) .
$$


The outcomes $x, y \in \Pi$ are payoff equivalent if $u(x)=u(y)$. The notion of payoff equivalence partitions the set of outcomes into equivalence classes. We denote the equivalence class containing $x$ by $[x]$. Kóczy and Lauwers (2007) say that the outcome $y \in X$ is accessible from the outcome $x \in X$ if $y \in g^{\mathbb{N}}(x)$ or $y$ is payoff equivalent to $x$. The set of outcomes that are accessible from $x$ in the sense of Kóczy and Lauwers (2007) can therefore be written as

$$
\widetilde{g^{\mathbb{N}}}(x)=g^{\mathbb{N}}(x) \cup[x] .
$$

The correspondence $\widetilde{g^{\mathbb{N}}}$ may fail to be transitive. As we are considering outcomes accessible from accessible outcomes it is natural to consider its transitive version:

$$
\widetilde{g}(x)=g(x) \cup[x] .
$$

We define the two-fold composition of $\widetilde{g}$ by $\widetilde{g}^{2}(x)=\{z \in X \mid \exists y \in \widetilde{g}(x)$ such that $z \in$ $\widetilde{g}(y)\}$. By induction, we define the $k$-fold iteration $\widetilde{g}^{k}(x)$ as the subset of $X$ that contains all outcomes obtained by a composition of dominance correspondences of length $k \in \mathbb{N}$, that is, $\widetilde{g}^{k}(x)=\left\{z \in X \mid \exists y \in \widetilde{g}^{k-1}(x)\right.$ such that $\left.z \in \widetilde{g}(y)\right\}$. Observe that for every $k, \ell \in \mathbb{N}$ if $k \leq \ell$, then $\widetilde{g}^{k}(x) \subseteq \widetilde{g}^{\ell}(x)$. We define the set of all outcomes that can be reached from $x$ by a finite number of dominations and payoff equivalences by $\widetilde{g}^{\mathbb{N}}(x)$, so

$$
\widetilde{g}^{\mathbb{N}}(x)=\bigcup_{k \in \mathbb{N}} \widetilde{g}^{k}(x) .
$$

It is easily seen that

$$
\widetilde{g}^{\mathbb{N}}(x)=(\widetilde{g})^{\mathbb{N}}(x) \supseteq \widetilde{g^{\mathbb{N}}}(x)=g^{\mathbb{N}}(x) \cup[x] .
$$

In this paper, we say that the outcome $y \in X$ is accessible from the outcome $x \in X$ if $y \in \widetilde{g}^{\mathbb{N}}(x)$.

Definition 2.3. Let $(N, v)$ be a coalition function form game. The set $\widetilde{\Delta} \subseteq X$ is a payoffequivalence minimal dominant set (PEMDS) if it satisfies the following three conditions:

1. Closure: For every $x \in \widetilde{\Delta}, \widetilde{g}^{\mathbb{N}}(x) \subseteq \widetilde{\Delta}$.

2. Accessibility: For every $x \notin \widetilde{\Delta}, \widetilde{g}^{\mathbb{N}}(x) \cap \widetilde{\Delta} \neq \emptyset$.

3. Minimality: There is no set $\widetilde{\Delta}^{\prime} \subsetneq \widetilde{\Delta}$ that satisfies Conditions 1 and 2 .

We argue next that multiple payoff-equivalence minimal dominant sets cannot co-exist. The argument relies heavily on the transitivity of $\widetilde{g}^{\mathbb{N}}$.

Theorem 2.4. A coalition function form game $(N, v)$ can have at most one PEMDS. 
Proof. Let $\widetilde{\Delta}_{1}$ and $\widetilde{\Delta}_{2}$ both be a PEMDS of $(N, v)$. We argue that $\widetilde{\Delta}_{1} \cap \widetilde{\Delta}_{2}$ satisfies Closure and Accessibility, which by Minimality implies that $\widetilde{\Delta}_{1}=\widetilde{\Delta}_{2}$.

To show Closure for $\widetilde{\Delta}_{1} \cap \widetilde{\Delta}_{2}$, let $x \in \widetilde{\Delta}_{1} \cap \widetilde{\Delta}_{2}$ and $y \in \widetilde{g}^{\mathbb{N}}(x)$. Then given that $x \in \widetilde{\Delta}_{1}$ and $\widetilde{\Delta}^{1}$ satisfies Closure, it must be that $y \in \widetilde{\Delta}_{1}$. Also given that $x \in \widetilde{\Delta}_{2}$ and $\widetilde{\Delta}_{2}$ satisfies closure, it must be that $y \in \widetilde{\Delta}_{2}$. This implies that $y \in \widetilde{\Delta}_{1} \cap \widetilde{\Delta}_{2}$ as was to be shown.

For Accessibility, take any $x \notin \widetilde{\Delta}_{1} \cap \widetilde{\Delta}_{2}$. There are three cases to consider.

Case 1. $x \in \widetilde{\Delta}_{1} \backslash \widetilde{\Delta}_{2}$. Then, by Accessibility of $\widetilde{\Delta}_{2}$, there is $y \in \widetilde{\Delta}_{2}$ such that $y \in \widetilde{g}^{\mathbb{N}}(x)$. By Closure of $\widetilde{\Delta}_{1}$, we have that $y \in \widetilde{\Delta}_{1}$. This means that $y \in \widetilde{\Delta}_{1} \cap \widetilde{\Delta}_{2}$, which is what we needed to show.

Case 2. $x \in \widetilde{\Delta}_{2} \backslash \widetilde{\Delta}_{1}$. The proof is symmetric to Case 1 with $\widetilde{\Delta}_{1}$ and $\widetilde{\Delta}_{2}$ interchanged.

Case 3. $x \in X \backslash\left(\widetilde{\Delta}_{1} \cup \widetilde{\Delta}_{2}\right)$. We know, by Accessibility of $\widetilde{\Delta}_{1}$, that there is $y \in \widetilde{\Delta}_{1}$ such that $y \in g^{\mathbb{N}}(x)$. If $y \in \widetilde{\Delta}_{2}$, we are done. If not, then we know from Case 1 that there is $z \in \widetilde{\Delta}_{1} \cap \widetilde{\Delta}_{2}$ such that $z \in \widetilde{g}^{\mathbb{N}}(y)$. It follows from transitivity of $\widetilde{g}^{\mathbb{N}}$ that $z \in \widetilde{g}^{\mathbb{N}}(x)$.

Kóczy and Lauwers (2007) also assert existence of a PEMDS for a coalition function form game $(N, v)$. Unfortunately, Kóczy and Lauwers (2007) do not use Accessibility consistently, for most of the paper the correspondence $g^{\mathbb{N}}$ is used interchangeably with $\widetilde{g^{\mathbb{N}}}$. Most notably Theorem 4 of Kóczy and Lauwers (2007) is false. The problematic assertion is that there exists a natural number $\tau \in \mathbb{N}$ such that for all outcomes $x, y \in X$ we have that $y \in \widetilde{g^{\mathbb{N}}}(x)$ if and only if $y \in g^{\tau}(x)$.

The following is a counterexample.

Example 2.5. Consider a game with $N=\{1,2\}$ and $v(C)=|C|$ for all $C \subseteq N$ and the outcome $x$ where $\mathcal{P}(x)=\{N\}$ and $u(x)=(1,1)$. This is an element of the coalition structure core CSC and therefore $g(x)=g^{\mathbb{N}}(x)=\{x\}$. On the other hand the outcome $y$ where $\mathcal{P}(y)=\langle N\rangle$ and $u(y)=u(x)$ is payoff-equivalent to $x$ and is therefore, by definition, accessible. Contrary to the assertion of the aforementioned Theorem, we have that $y \in$ $\widetilde{g^{\mathbb{N}}}(x) \subseteq \widetilde{g}^{\mathbb{N}}(x)$, whereas $y \notin g^{\mathbb{N}}(x)$.

\section{The Minimal Dominant Set}

It is clear that payoff-equivalence causes problems, while it turns out that it is completely superfluous and can be eliminated. In this section we drop pay-off equivalence from the dominance correspondence. Other papers in the literature studying the payoff-equivalence minimal dominant set have done so as well, see for instance Yang (2011, 2020). This makes also sense from a conceptual point of view. For a new coalition to form, at least one of the coalition members should improve, which is not the case under payoff-equivalence. 
Definition 3.1. Let $(N, v)$ be a coalition function form game. The set $\Delta \subseteq X$ is a minimal dominant set $(M D S)$ if it satisfies the following three conditions:

1. Closure: For every $x \in \Delta, g^{\mathbb{N}}(x) \subseteq \Delta$.

2. Sequential weak dominance: For every $x \notin \Delta, g^{\mathbb{N}}(x) \cap \Delta \neq \emptyset$.

3. Minimality: There is no set $\Delta^{\prime} \subsetneq \Delta$ that satisfies Conditions 1 and 2 .

Definition 3.1 is obtained from Definition 2.3 by replacing the correspondence $\widetilde{g}^{\mathbb{N}}$ by $g^{\mathbb{N}}$. The only difference between these correspondences is the absence of payoff-equivalence in the latter, so for every $x \in X$ it holds that $g^{\mathbb{N}}(x) \subseteq \widetilde{g}^{\mathbb{N}}(x)$.

The proof of Theorem 4 of Kóczy and Lauwers (2007) relies on a construction where the set of outcomes are partitioned into similarity classes. Similarity classes are closed and convex sets of outcomes determined by the coalitional deviations they are subject to. Notably, two distinct, payoff-equivalent outcomes belong to different similarity classes and their payoff-equivalence is not taken into account at all. As a result, if we replace Accessibility with Sequential weak dominance, Theorem 4 of Kóczy and Lauwers (2007) holds.

Theorem 3.2. Let $(N, v)$ be a game. Then there exists a natural number $\tau \in \mathbb{N}$ such that for all outcomes $x, y \in X$ we have that $y \in g^{\mathbb{N}}(x)$ if and only if $y \in g^{\tau}(x)$.

While this result presents no explicit bounds, Yang (2011) shows that elements of the MDS can be reached in a number of steps that is quadratic in $n$.

Next, we can proceed as in Kóczy and Lauwers (2007) to show the following result.

Theorem 3.3. A coalition function form game $(N, v)$ has a unique MDS. If the coalition structure core of the game is non-empty, then it is equal to the MDS.

It follows from a counterexample in Bhattacharya and Ziad (2006) that Theorem 3.3 cannot be extended to the non-transferable utility case; Kóczy (2018, p 99) conjectures the same for partition function form games.

What is the effect of the removal of payoff equivalence on the solution concept? Note that for a generic coalition function form game $(N, v)$, if $\mathcal{P}, \mathcal{Q} \in \Pi$ are different partitions of $N$, so $\mathcal{P} \neq \mathcal{Q}$, then $\sum_{C \in \mathcal{P}} v(C) \neq \sum_{C \in \mathcal{Q}} v(C)$.

Theorem 3.4. Let $(N, v)$ be a coalition function form game such that, for every $\mathcal{P}, \mathcal{Q} \in \Pi$ such that $\mathcal{P} \neq \mathcal{Q}, \sum_{C \in \mathcal{P}} v(C) \neq \sum_{C \in \mathcal{Q}} v(C)$. Then $\Delta$ is an $\operatorname{MDS}$ of $(N, v)$ if and only if it is a PEMDS of $(N, v)$.

Proof. The assumption of Theorem 3.4 implies that payoff equivalences cannot occur. Therefore, it holds that $g^{\mathbb{N}}=\widetilde{g}^{\mathbb{N}}$. 
Theorem 3.4 establishes that for generic coalition function form games, the PEMDS coincides with the MDS.

Apart from the generic case where multiple partitions with the same total payoff do not exist, there is another interesting class of coalition function form games where the predictions of PEMDS and MDS coincide, that is, coalition function form games with a non-empty coalition structure core.

Theorem 3.5. Let $(N, v)$ be a coalition function form game with a non-empty coalition structure core. Then the PEMDS of $(N, v)$ is equal to the coalition structure core.

Proof. We argue first that any element of the coalition structure core belongs to a PEMDS. Assume that $\widetilde{\Delta}$ is a PEMDS. Let $x \in$ CSC. It holds that $g^{\mathbb{N}}(x)=\{x\}$. For every $y \in[x]$, it holds that $y$ belongs to CSC, so $g^{\mathbb{N}}(y)=\{y\}$ and therefore $\widetilde{g}^{\mathbb{N}}(x)=[x]$.

Suppose $x \notin \widetilde{\Delta}$. Then, we have $\widetilde{g}^{\mathbb{N}}(x) \cap \widetilde{\Delta}=[x] \cap \widetilde{\Delta} \neq \emptyset$. Closure of $\widetilde{\Delta}$ now implies $[x] \subseteq \widetilde{\Delta}$, contradicting $x \notin \widetilde{\Delta}$. Consequently, it holds that $x \in \widetilde{\Delta}$.

We argue that CSC satisfies Closure and Accessibility, which by Minimality implies that $\widetilde{\Delta}=$ CSC. We already showed that for every $x \in \mathrm{CSC}$ we have $\widetilde{g}^{\mathbb{N}}(x)=[x]$. This shows Closure.

By Theorem 3.3, it holds that the MDS of $(N, v)$ is equal to CSC, so CSC satisfies Sequential weak dominance and therefore Accessibility.

It follows from Theorems 3.3 and 3.5 that the MDS and the PEMDS coincide for coalition function form games with a non-empty coalition structure core.

We now present an example where the MDS is a proper subset of the PEMDS.

Example 3.6. Let $(N, v)$ be the three-player simple majority game, so $N=\{1,2,3\}$ and $v(\{1,2\})=v(\{1,3\})=v(\{2,3\})=v(N)=1$, whereas the worth of any other coalition is equal to zero. It holds that $\mathrm{CSC}=\emptyset$. Let $\mathcal{M}=\{\{1,2\},\{1,3\},\{2,3\}\}$ be the set of minimal winning coalitions. Consider the set $F \subseteq X$ defined by

$$
F=\{x \in X \mid \mathcal{P}(x) \cap \mathcal{M} \neq \emptyset\}
$$

so in payoff terms the set $F$ is given by the facets of the unit simplex. It is easily verified that $F$ satisfies Closure with respect to $g^{\mathbb{N}}$ and Accessibility. Moreover, for every $x \in F$, it holds that $g^{\mathbb{N}}(x)=F$, so there is no proper subset of $F$ that satisfies Closure with respect to $g^{\mathbb{N}}$ and Accessibility. It follows that the MDS of $(N, v)$ is equal to $F$. The prediction of the MDS is that a minimal winning coalition forms and one player becomes a singleton. It is excluded that the grand coalition forms.

The set $F$ is not a PEMDS, since outcomes which are payoff equivalent to outcomes in $F$ may not belong to $F$ themselves. For instance, for every $x \in F$ it holds that $y \in X$ 
defined by $\mathcal{P}(y)=\{N\}$ and $u(y)=u(x)$ is payoff equivalent to $x$, but is not part of $F$. We define the set $\widetilde{F} \subseteq X$ by

$$
\begin{aligned}
\widetilde{F} & =F \cup\left\{x \in X \mid \mathcal{P}(x)=\{N\} \text { and there exists } i \in N \text { such that } u_{i}(x)=0\right\} \\
& =\left\{x \in X \mid \mathcal{P}(x) \cap(\mathcal{M} \cup\{N\}) \neq \emptyset \text { and there exists } i \in N \text { such that } u_{i}(x)=0\right\} .
\end{aligned}
$$

It is easily verified that the PEMDS of $(N, v)$ is given by $\widetilde{F}$.

Example 3.6 presents an example where the PEMDS is different from the MDS, but the same when projected to the space of payoffs. This turns out to be a general feature of so-called proper simple games as we will formally state in Theorem 5.5. It is natural to ask if the property holds in general: should we ignore partitions and focus on the payoff vectors only, do we have equivalence? The next example answers this question in the negative.

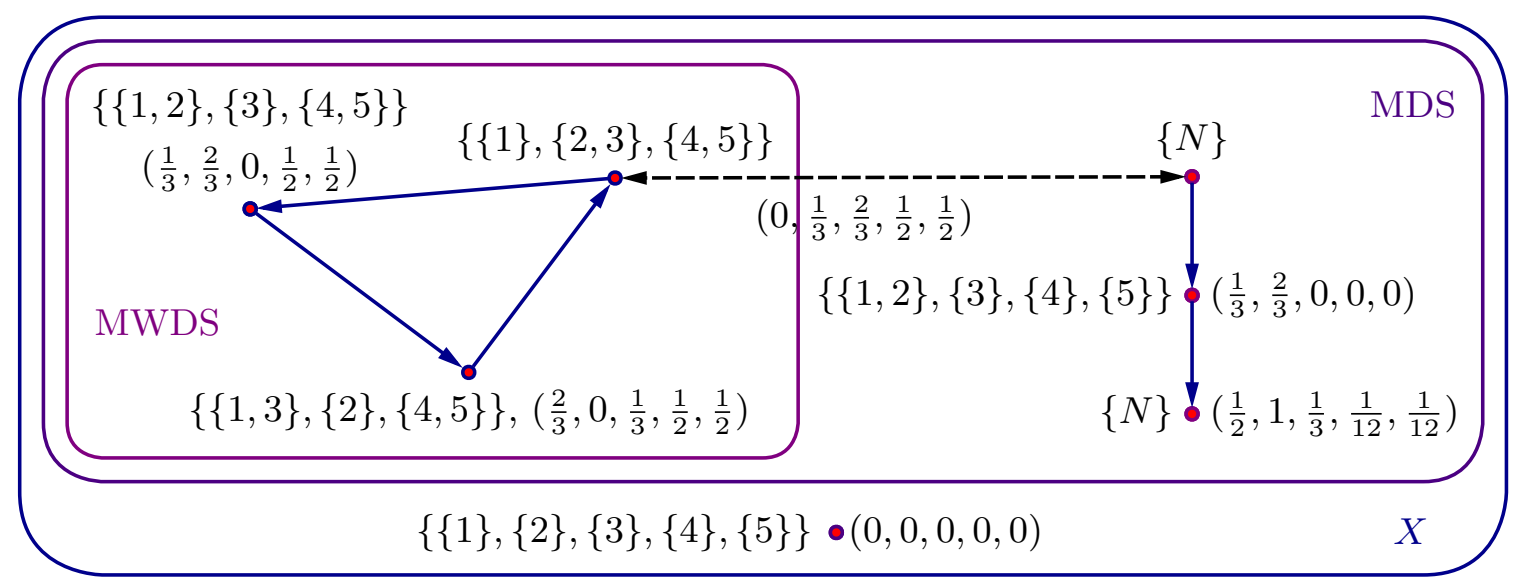

Figure 1: An illustration of Example 3.7. Arrows indicate weak dominances, dashed lines payoff equivalence. Not all weak dominances are displayed.

Example 3.7. Consider the following 5-player example. Coalitions $\{1,2\},\{1,3\}$ and $\{2,3\}$ have a worth of 1 ; so does coalition $\{4,5\}$. The grand coalition $\{1,2,3,4,5\}$ has a worth of 2 .

The MDS equals the union of the facets of the simplex on players $\{1,2,3\}$, with one of the two-player subsets forming as a coalition, plus an arbitrary payoff division of the worth of 1 of coalition $\{4,5\}$. The grand coalition does not have a profitable deviation from this, so is not part of the MDS. On the other hand, it is part of a PEMDS $\widetilde{\Delta}$ by payoff equivalence. Now at least one of the coalitions $\{1,2\},\{1,3\}$, or $\{2,3\}$ can deviate, and players 4 and 5 become singletons. Now the grand coalition can deviate with a payoff vector different from any payoff vector in the MDS. One can show that the PEMDS contains much more of the outcomes although not all - with one trivial example indicated in Figure 1. 


\section{The Myopic Stable Set}

Demuynck, Herings, Saulle, and Seel (2019) introduce the solution concept of the myopic stable set for general social environments. They then use the concept to study specific social environments coming from applications like coalition function form games, matching models, networks, and non-cooperative games. We now explain how the solution concept is defined when applied to coalition function form games.

Definition 4.1. An outcome $y \in X$ dominates an outcome $x \in X$ by deviating coalition $D$ if

- $\mathcal{P}(y)=\{D\} \cup\langle R(D, \mathcal{P}(x))\rangle \cup \mathcal{O}(D, \mathcal{P}(x))$,

and

- for every $i \in D, u_{i}(y)>u_{i}(x)$,

- for every $i \in O(D, \mathcal{P}(x)), u_{i}(y)=u_{i}(x)$.

The only difference between Definitions 2.1 and 4.1 is that the condition that at least one member of the deviating coalition strictly improves has been replaced by the requirement that all members of the deviating coalition strictly improve.

Let some outcome $x \in X$ be given. The subset of $X$ consisting of all outcomes that dominate $x$ together with outcome $x$ itself is denoted by $f(x)$, so

$$
f(x)=\{x\} \cup\{y \in X \mid \text { there is } D \in \mathcal{N} \text { such that } y \text { dominates } x \text { by } D\} \text {. }
$$

As before, we define the two-fold composition of $f$ by $f^{2}(x)=\{z \in X \mid \exists y \in f(x)$ such that $z \in$ $f(y)\}$. By induction, we define the $k$-fold iteration $f^{k}(x)$ as the subset of $X$ that contains all outcomes obtained by a composition of dominance correspondences of length $k \in \mathbb{N}$, that is, $f^{k}(x)=\left\{z \in X \mid \exists y \in f^{k-1}(x)\right.$ such that $\left.z \in f(y)\right\}$. Observe that for every $k, \ell \in \mathbb{N}$ if $k \leq \ell$, then $f^{k}(x) \subseteq f^{\ell}(x)$. We define the set of all outcomes that can be reached from $x$ by a finite number of dominations by $f^{\mathbb{N}}(x)$, so $f^{\mathbb{N}}(x)=\bigcup_{k \in \mathbb{N}} f^{k}(x)$.

An outcome $y$ is said to asymptotically dominate the outcome $x$ if starting from $x$, it is possible to get arbitrarily close to $y$ in a finite number of dominations. We define the metric $d$ on $X$ by setting for every $x, y \in X$,

$$
d(x, y)=\mathbb{1}_{\{\mathcal{P}(x) \neq \mathcal{P}(y)\}}+\|u(x)-u(y)\|_{\infty},
$$

where $\mathbb{1}$ is the indicator function and $\|\cdot\|_{\infty}$ is the infinity norm.

Notice that payoff-equivalent outcomes with different underlying partitions are not near due to the first term of the metric. 
Definition 4.2. An outcome $y \in X$ asymptotically dominates an outcome $x \in X$ if for every $\varepsilon>0$ there exists a number $k \in \mathbb{N}$ and an outcome $z \in f^{k}(x)$ such that $d(y, z)<\varepsilon$.

We denote by $f^{\infty}(x)$ the set of all outcomes in $X$ that asymptotically dominate $x$. Formally,

$$
f^{\infty}(x)=\left\{y \in X \mid \forall \varepsilon>0, \exists k \in \mathbb{N}, \exists z \in f^{k}(x): d(y, z)<\varepsilon\right\}
$$

It is easy to see that the set $f^{\infty}(x)$ coincides with the closure of the set $f^{\mathbb{N}}(x)$.

We are now ready to define the myopic stable set, abbreviated as MSS.

Definition 4.3. Let $(N, v)$ be a coalition function form game. The set $M \subseteq X$ is a myopic stable set (MSS) if it is closed and satisfies the following three conditions:

1. Deterrence of external deviations: For every $x \in M, f(x) \subseteq M$.

2. Asymptotic external stability: For every $x \notin M, f^{\infty}(x) \cap M \neq \emptyset$.

3. Minimality: There is no closed set $M^{\prime} \subsetneq M$ that satisfies Conditions 1 and 2 .

Let $M$ be an MSS. Deterrence of external deviations requires that no coalition can profitably deviate to an outcome outside $M$. Asymptotic external stability requires that any outcome outside $M$ is asymptotically dominated by an outcome in $M$. Hence, from any outcome outside of $M$ it is possible to get arbitrary close to an outcome in $M$ by a finite number of dominations. Observe that an empty set would necessarily violate external stability, so any MSS is non-empty. Minimality imposes that there is no smaller closed set of outcomes that satisfies Deterrence of external deviations and Asymptotic external stability.

Apart from the use of the dominance correspondence instead of the weak dominance correspondence, there are three differences between the MSS and the MDS. The requirement of Closure for MDS has been replaced by Deterrence of external deviations in MSS. Since "for every $x \in M, f(x) \subseteq M$ " implies "for every $x \in M, f^{\mathbb{N}}(x) \subseteq M$," this difference reduces to the use of the dominance correspondence instead of the weak dominance correspondence. The second difference is that the requirement of Sequential weak dominance in MDS is based on the finitely iterated dominance correspondence, whereas Asymptotic external stability in MSS is based on the asymptotic dominance correspondence. Finally, the MSS is a closed set by definition.

Demuynck, Herings, Saulle, and Seel (2019) show the following result.

Theorem 4.4. A coalition function form game $(N, v)$ has a unique MSS. If the coalition structure core of the game is non-empty, then it is equal to the MSS. 
As a consequence of Theorems 3.3, 3.5, and 4.4, we conclude that the MDS, the PEMDS, and the MSS coincide whenever CSC $\neq \emptyset$. Example 3.6 shows that in general MDS and PEMDS are not the same. We next argue that in Example 3.6, the MSS coincides with the MDS.

Example 4.5. As in Example 3.6, let $(N, v)$ be the three-player simple majority game, so $N=\{1,2,3\}$ and $v(\{1,2\})=v(\{1,3\})=v(\{2,3\})=v(N)=1$, whereas the worth of any other coalition is equal to zero. Recall that $\mathcal{M}=\{\{1,2\},\{1,3\},\{2,3\}\}$ is the set of minimal winning coalitions. It follows from Theorem 4.5 in Demuynck, Herings, Saulle, and Seel (2019) that the MSS is equal to the set

$$
F=\{x \in X \mid \mathcal{P}(x) \cap \mathcal{M} \neq \emptyset\}
$$

so the MSS coincides with the MDS.

The equivalence of the MSS and the MDS holds in general; this is the topic of the next section.

\section{Equivalence}

Before showing the relation between the MSS and MDS, we first introduce another concept, the weak dominance MSS, which results from replacing the dominance correspondence in the MSS by the weak dominance correspondence.

Definition 5.1. Let $(N, v)$ be a coalition function form game. The set $W \subseteq X$ is a weak dominance myopic stable set (weak dominance MSS) if it is closed and satisfies the following three conditions:

1. Deterrence of weak external deviations: For every $x \in W, g(x) \subseteq W$.

2. Asymptotic weak external stability: For every $x \notin W, g^{\infty}(x) \cap W \neq \emptyset$.

3. Minimality: There is no closed set $W^{\prime} \subsetneq W$ that satisfies Conditions 1 and 2 .

The weak dominance MSS has also been studied in Demuynck et al. (2019) and it is explained there how most results established for the MSS carry over to the weak dominance MSS.

We show first that the concepts of MSS and weak dominance MSS are equivalent.

To do so, we need two lemmas first. The first one makes clear that Asymptotic external stability, Asymptotic weak external stability, and Sequential weak dominance are equivalent. 
Lemma 5.2. Let $(N, v)$ be a coalition function form game. For every $x \in X$ it holds that $f^{\infty}(x)=g^{\infty}(x)=g^{\mathbb{N}}(x)$.

Proof. Let $x \in X$.

Lemma 9 in Kóczy and Lauwers (2007) states that $g^{\mathbb{N}}(x)$ is a closed set, so $g^{\infty}(x)=$ $g^{\mathbb{N}}(x)$.

Since $f(x) \subseteq g(x)$, it follows immediately that $f^{\infty}(x) \subseteq g^{\infty}(x)=g^{\mathbb{N}}(x)$.

To show the reverse inclusion, let some $y \in g^{\mathbb{N}}(x)$ be given, so there exists a sequence of outcomes $\left(x^{0}, \ldots, x^{\ell}\right)$ such that, for every $k=1, \ldots, \ell, x^{k} \in f\left(x^{k-1}\right)$. For $k=1, \ldots, \ell$, let $D^{k}$ be the deviating coalition that changes the outcome from $x^{k-1}$ to $x^{k}$ and $P^{k}=$ $P\left(D^{k}, \mathcal{P}\left(x^{k-1}\right)\right)$ be the partners' set of $D^{k}$ in $\mathcal{P}\left(x^{k-1}\right)$. We also define $R^{k}=P^{k} \backslash D^{k}$ and $O^{k}=N \backslash P^{k}$ as the set of residual players and outsiders, respectively. We have that

$$
\begin{array}{ll}
u_{i}\left(x^{k}\right)=v(\{i\}), & i \in R^{k}, \\
u_{i}\left(x^{k}\right)=u_{i}\left(x^{k-1}\right), & i \in O^{k} .
\end{array}
$$

We define $W^{k} \subset D^{k}$ to be the, possibly empty, proper subset of $D^{k}$ consisting of players that only weakly improve when moving from outcome $x^{k-1}$ to outcome $x^{k}$, so for every $i \in W^{k}$ it holds that $u_{i}\left(x^{k-1}\right)=u_{i}\left(x^{k}\right)$. We define

$$
\begin{aligned}
\delta & =\min _{k \in\{1, \ldots, \ell\}} \min _{i \in D^{k} \backslash W^{k}} u_{i}\left(x^{k}\right)-u_{i}\left(x^{k-1}\right), \\
\varepsilon^{\prime} & =\min \{\delta, \varepsilon\}
\end{aligned}
$$

so $\delta$ is the smallest improvement of any of the strictly improving players involved in any move along the sequence. It holds that $\delta>0$ and therefore that $\varepsilon^{\prime}>0$. For $k \in\{0, \ldots, \ell\}$, define

$$
\nu_{k}=\frac{n^{2 k}}{n^{2 \ell+1}} .
$$

We define $e\left(W^{k}\right)=0$ if $W^{k}=\emptyset$ and $e\left(W^{k}\right)=1$ otherwise. We use the sequence $\left(x^{0}, x^{1}, \ldots, x^{\ell}\right)$ of outcomes to define a new sequence $\left(\widetilde{x}^{0}, \widetilde{x}^{1}, \ldots, \widetilde{x}^{\ell}\right)$ of outcomes by setting $\widetilde{x}^{0}=x^{0}$ and, for every $k \in\{1, \ldots, \ell\}$,

$$
\begin{aligned}
& \mathcal{P}\left(\widetilde{x}^{k}\right)= \\
& u_{i}\left(\widetilde{x}^{k}\right)= \begin{cases}u_{i}\left(x^{k}\right)+\varepsilon^{\prime} \nu_{k} \frac{\left|D^{k} \backslash W^{k}\right|}{\left|W^{k}\right|}, & i \in W^{k}, \\
u_{i}\left(x^{k}\right)-\varepsilon^{\prime} \nu_{k} e\left(W^{k}\right), & i \in D^{k} \backslash W^{k}, \\
u_{i}\left(x^{k}\right)=v(\{i\}), & i \in R^{k}, \\
u_{i}\left(\widetilde{x}^{k-1}\right), & i \in O^{k} .\end{cases}
\end{aligned}
$$

Notice that the first line does not entail a division by zero, since if $i \in W^{k}$, then $W^{k} \neq \emptyset$. 
Compared to the sequence $\left(x^{0}, x^{1}, \ldots, x^{\ell}\right)$, the sequence $\left(\widetilde{x}^{0}, \widetilde{x}^{1}, \ldots, \widetilde{x}^{\ell}\right)$ is such that each strictly improving player in $D^{k} \backslash W^{k}$ donates an amount $\varepsilon^{\prime} \nu_{k} /\left|W^{k}\right|$ to each of the players in $W^{k}$ whenever the latter set is non-empty. It is also important to observe that the fraction $\nu_{k}$ is an $n^{2}$ multiple of $\nu_{k-1}$ and that $\nu_{\ell}=1 / n$.

We show first by induction that, for every $k \in\{0, \ldots, \ell\}, \widetilde{x}^{k} \in X$. Obviously, it holds that $\widetilde{x}^{0}=x^{0} \in X$. Assume that, for some $k \in\{1, \ldots, \ell\}, \widetilde{x}^{k-1} \in X$. We show that $\widetilde{x}^{k} \in X$. It holds that

$$
\begin{aligned}
& u_{i}\left(\widetilde{x}^{k}\right)>u_{i}\left(x^{k}\right) \geq v(\{i\}), \quad i \in W^{k}, \\
& u_{i}\left(\widetilde{x}^{k}\right) \geq u_{i}\left(x^{k-1}\right)+\delta-\varepsilon^{\prime} \nu_{k}>u_{i}\left(x^{k-1}\right)+\delta-\varepsilon^{\prime} \geq u_{i}\left(x^{k-1}\right) \geq v(\{i\}), \quad i \in D^{k} \backslash W^{k}, \\
& u_{i}\left(\widetilde{x}^{k}\right)=v(\{i\}), \quad i \in R^{k} \text {, } \\
& u_{i}\left(\widetilde{x}^{k}\right)=u_{i}\left(\widetilde{x}^{k-1}\right) \geq v(\{i\}), \quad i \in O^{k},
\end{aligned}
$$

where the very last inequality follows from the induction hypothesis. Moreover, for every $C \in \mathcal{P}\left(x^{k}\right)$, it holds that either $C=D^{k}$ and $W^{k}=\emptyset$, so

$$
\sum_{i \in C} u_{i}\left(\widetilde{x}^{k}\right)=\sum_{i \in D^{k}} u_{i}\left(x^{k}\right)=v(C),
$$

or $C=D^{k}$ and $W^{k} \neq \emptyset$, so

$$
\sum_{i \in C} u_{i}\left(\widetilde{x}^{k}\right)=\sum_{i \in W^{k}}\left(u_{i}\left(x^{k}\right)+\varepsilon^{\prime} \nu_{k} \frac{\left|D^{k} \backslash W^{k}\right|}{\left|W^{k}\right|}\right)+\sum_{i \in D^{k} \backslash W^{k}}\left(u_{i}\left(x^{k}\right)-\varepsilon^{\prime} \nu_{k}\right)=\sum_{i \in D^{k}} u_{i}\left(x^{k}\right)=v(C),
$$

or $C=\left\{i^{\prime}\right\}$ with $i^{\prime} \in R^{k}$ and

$$
\sum_{i \in C} u_{i}\left(\widetilde{x}^{k}\right)=u_{i^{\prime}}\left(\widetilde{x}^{k}\right)=u_{i^{\prime}}\left(x^{k}\right)=v\left(\left\{i^{\prime}\right\}\right)=v(C),
$$

or $C \subseteq O^{k}$, so $C \in \mathcal{P}\left(\widetilde{x}^{k-1}\right)$, and

$$
\sum_{i \in C} u_{i}\left(\widetilde{x}^{k}\right)=\sum_{i \in C} u_{i}\left(\widetilde{x}^{k-1}\right)=v(C)
$$

where the last equality makes use of the induction hypothesis. We have now completed the proof of the fact that for every $k \in\{0, \ldots, \ell\}, \widetilde{x}^{k} \in X$.

We show next by induction that, for every $k \in\{0, \ldots, \ell\}$, and for every $i \in N$,

$$
\left|u_{i}\left(\widetilde{x}^{k}\right)-u_{i}\left(x^{k}\right)\right| \leq \varepsilon^{\prime} \nu_{k}(n-1) .
$$

Obviously, for every $i \in N$, it holds that $\left|u_{i}\left(\widetilde{x}^{0}\right)-u_{i}\left(x^{0}\right)\right|=0 \leq \varepsilon^{\prime} \nu_{0}(n-1)$. Assume that, for some $k \in\{1, \ldots, \ell\}$, for every $i \in N,\left|u_{i}\left(\widetilde{x}^{k-1}\right)-u_{i}\left(x^{k-1}\right)\right| \leq \varepsilon^{\prime} \nu_{k-1}(n-1)$. We show 
that, for every $i \in N,\left|u_{i}\left(\widetilde{x}^{k}\right)-u_{i}\left(x^{k}\right)\right| \leq \varepsilon^{\prime} \nu_{k}(n-1)$. If $i \in W^{k}$, then $W^{k} \neq \emptyset$, and the statement follows from the observation that

$$
0 \leq u_{i}\left(\widetilde{x}^{k}\right)-u_{i}\left(x^{k}\right)=\varepsilon^{\prime} \nu_{k} \frac{\left|D^{k} \backslash W^{k}\right|}{\left|W^{k}\right|} \leq \varepsilon^{\prime} \nu_{k}(n-1) .
$$

If $i \in D^{k} \backslash W^{k}$, then we have that

$$
0 \geq u_{i}\left(\widetilde{x}^{k}\right)-u_{i}\left(x^{k}\right) \geq-\varepsilon^{\prime} \nu_{k} \geq-\varepsilon^{\prime} \nu_{k}(n-1) .
$$

If $i \in R^{k}$, then $\left|u_{i}\left(\widetilde{x}^{k}\right)-u_{i}\left(x^{k}\right)\right|=0$. If $i \in O^{k}$, then it holds that

$$
\left|u_{i}\left(\widetilde{x}^{k}\right)-u_{i}\left(x^{k}\right)\right|=\left|u_{i}\left(\widetilde{x}^{k-1}\right)-u_{i}\left(x^{k-1}\right)\right| \leq \varepsilon^{\prime} \nu_{k-1}(n-1)<\varepsilon^{\prime} \nu_{k}(n-1),
$$

where the first inequality makes use of the induction hypothesis and the last inequality of the fact that $\nu_{k-1}<\nu_{k}$.

Let some $k \in\{1, \ldots, \ell\}$ and some $i \in D^{k}$ be given. We show that $u_{i}\left(\widetilde{x}^{k}\right)>u_{i}\left(\widetilde{x}^{k-1}\right)$. If $i \in W^{k}$, then it holds that

$$
\begin{aligned}
u_{i}\left(\widetilde{x}^{k}\right) & =u_{i}\left(x^{k}\right)+\varepsilon^{\prime} \nu_{k} \frac{\left|D^{k} \backslash W^{k}\right|}{\left|W^{k}\right|} \\
& =u_{i}\left(x^{k-1}\right)+\varepsilon^{\prime} \nu_{k} \frac{\left|D^{k} \backslash W^{k}\right|}{\left|W^{k}\right|} \\
& \geq u_{i}\left(\tilde{x}^{k-1}\right)-\varepsilon^{\prime} \nu_{k-1}(n-1)+\varepsilon^{\prime} \nu_{k} \frac{1}{n-1} \\
& >u_{i}\left(\widetilde{x}^{k-1}\right)
\end{aligned}
$$

where the strict inequality uses that $\nu_{k}=n^{2} \nu_{k-1}$. If $i \in D^{k} \backslash W^{k}$, then it holds that

$$
\begin{aligned}
u_{i}\left(\widetilde{x}^{k}\right) & \geq u_{i}\left(x^{k}\right)-\varepsilon^{\prime} \nu_{k} \\
& \geq u_{i}\left(x^{k-1}\right)+\delta-\varepsilon^{\prime} \nu_{k} \\
& \geq u_{i}\left(\widetilde{x}^{k-1}\right)-\varepsilon^{\prime} \nu_{k-1}(n-1)+\delta-\varepsilon^{\prime} n^{2} \nu_{k-1} \\
& >u_{i}\left(\widetilde{x}^{k-1}\right)
\end{aligned}
$$

where the strict inequality uses the facts that $\delta \geq \varepsilon^{\prime}$ and

$$
\left(n^{2}+(n-1)\right) \nu_{k-1}<2 n^{2} \nu_{k-1} \leq 2 \nu_{k} \leq 1 \text {. }
$$

Combining the statements proven so far, it follows that $\widetilde{x}^{\ell} \in f^{\ell}\left(x^{0}\right)$.

The proof is completed by observing that $d\left(\widetilde{x}^{\ell}, x^{\ell}\right)<\varepsilon$ since $\mathcal{P}\left(\widetilde{x}^{\ell}\right)=\mathcal{P}\left(x^{\ell}\right)$ and, for every $i \in N$,

$$
\left|u_{i}\left(\widetilde{x}^{\ell}\right)-u_{i}\left(x^{\ell}\right)\right| \leq \varepsilon^{\prime} \nu_{\ell}(n-1)<\varepsilon^{\prime} \leq \varepsilon .
$$


In the proof of Theorem 4.4 in Demuynck, Herings, Saulle, and Seel (2019) it has been shown that any element in CSC belonging to $g^{\mathbb{N}}(x)$ is contained in $f^{\infty}(x)$. The same proof technique is used in the proof of Lemma 4.2 to extend this result and show that this does not only apply to coalition structure core elements, but holds in general.

Example A.6 of the online supplement of Demuynck, Herings, Saulle, and Seel (2019) considers a coalition function form game such that $N=\{1,2,3\}, v(\{1,2\})=1$, and $v(\{2,3\})=1$. All other coalitions have a worth of 0 . Thus, player 2 can choose between player 1 and player 3 to form a two-person coalition generating a surplus equal to one. It holds that CSC consists of only two states, $y$ and $y^{\prime}$, with equal payoffs, $u(y)=u\left(y^{\prime}\right)=$ $(0,1,0)$, and coalitional structures $\mathcal{P}(y)=\{\{1,2\},\{3\}\}$ and $\mathcal{P}\left(y^{\prime}\right)=\{\{1\},\{2,3\}\}$.

Consider an initial state $x^{0} \in X$ such that $\mathcal{P}\left(x^{0}\right)=\{\{1\},\{2\},\{3\}\}$ and $u\left(x^{0}\right)=(0,0,0)$. It can be shown that any element $x^{1}$ of $f^{\mathbb{N}}\left(x^{0}\right)$ is such that either $\mathcal{P}\left(x^{1}\right)=\{\{1,2\},\{3\}\}$ and $u\left(x^{1}\right)=(\varepsilon, 1-\varepsilon, 0)$ for some $\varepsilon \in(0,1)$ or $\mathcal{P}\left(x^{1}\right)=\{\{1\},\{2,3\}\}$ and $u\left(x^{1}\right)=(0,1-\varepsilon, \varepsilon)$ for some $\varepsilon \in(0,1)$. In particular, it holds that $f^{\mathbb{N}}\left(x^{0}\right)$ does not contain an element of CSC. On the contrary, it is immediately verified that both coalition structure core elements belong to $g\left(x^{0}\right)$. It therefore holds that $f^{\mathbb{N}}\left(x^{0}\right)$ is not closed. The example shows that $f^{\mathbb{N}}\left(x^{0}\right)$ is a proper subset of $g^{\mathbb{N}}\left(x^{0}\right)$. It is therefore impossible to extend Lemma 5.2 and obtain an equivalence with $f^{\mathbb{N}}(x)$ as well.

Theorem 5.3. A coalition function form game $(N, v)$ has a unique weak dominance MSS. It coincides with the MSS.

Proof. Let $Y \subseteq X$ be any closed set of outcomes that satisfies Deterrence of external deviations and Asymptotic external stability. We show that it satisfies Deterrence of weak external deviations and Asymptotic weak external stability. The latter property follows immediately from Lemma 5.2.

Suppose there is $y \in Y$ such that $g(y) \backslash Y \neq \emptyset$. Let $z \in g(y) \backslash Y$ and $D \in \mathcal{N}$ be such that $z$ weakly dominates $y$ by $D$. Let $j \in D$ be such that $u_{j}(z)>u_{j}(y)$. If $u_{i}(z)>u_{i}(y)$ for every $i \in D$, then $z \in f(y) \backslash Y$, contradicting that $Y$ satisfies Deterrence of external deviations. It follows that there is at least one $i \in D$ such that $u_{i}(z)=u_{i}(y)$.

Since $Y$ is closed and $z \notin Y$, there is $\varepsilon>0$ such that, for every $y \in Y, d(y, z)>\varepsilon$. Moreover, $\varepsilon$ can be taken sufficiently small to satisfy $\varepsilon<z_{j}-y_{j}$. We define $\widetilde{z} \in X$ by

$$
\begin{array}{lll}
\widetilde{z}_{j}=z_{j}-\varepsilon, & \\
\widetilde{z}_{i}=z_{i}+\varepsilon /(|D|-1), & & i \in D \backslash\{j\}, \\
\widetilde{z}_{i}=z_{i}, & & i \in N \backslash D .
\end{array}
$$

It is easily verified that $\widetilde{z} \in f(y)$. Since $d(z, \widetilde{z})=\varepsilon$, it holds that $\widetilde{z} \notin Y$. We have obtained a contradiction with the fact that $Y$ satisfies Deterrence of external deviations. Consequently, 
it holds for every $y \in Y$ that $g(y) \subseteq Y$, that is $Y$ satisfies Deterrence of weak external deviations.

Since any closed set of outcomes that satisfies Deterrence of external deviations and Asymptotic external stability also satisfies Deterrence of weak external deviations and Asymptotic weak external stability, we have that the weak dominance MSS coincides with the MSS and is therefore unique by Theorem 3.4.

After having shown the equivalence of the MSS and the weak dominance MSS, we turn to the relationship between the MSS and the MDS.

Theorem 5.4. The MSS and the MDS of a coalition function form game $(N, v)$ coincide.

Proof. We show that the three conditions of Definitions 3.1 and 5.1 are equivalent and that the MDS is a closed set, after which the result follows from Theorem 5.3.

Consider some set $Y \subseteq X$. The statement "for every $x \in Y, g(x) \subseteq Y$ " implies the statement "for every $x \in Y, g^{\mathbb{N}}(x) \subseteq Y$." Conversely, we have that for every $x \in Y$, " $g^{\mathbb{N}}(x) \subseteq Y$ " implies " $g^{1}(x)=g(x) \subseteq Y$." Therefore, Closure and the Deterrence of weak external deviations are equivalent.

By Lemma 5.2 Asymptotic external stability is equivalent to Sequential weak dominance, therefore $g^{\infty}(x) \cap Y \neq \emptyset$ is equivalent to $g^{\mathbb{N}}(x) \cap Y \neq \emptyset$.

At last, Theorem 19 in Kóczy and Lauwers (2007) states that the MDS can be written as a finite union of sets of outcomes called similarity classes. Each similarity class is defined by a finite number of linear weak inequalities and is therefore closed. This implies that the MDS is closed. The MDS is the smallest set satisfying Closure and Sequential weak dominance, but then it is also the smallest closed set satisfying these properties. This completes the proof.

The result of Theorems 5.3 and 5.4 imply that MDS, MSS, and weak dominance MSS coincide for all coalition function form games. Moreover, they also coincide with the PEMDS for important cases like generic coalition function form games (Theorem 3.4) and coalition function form games with a non-empty coalition structure core (Theorem 3.5). Example 3.6 shows that the coincide with PEMDS is not true in terms of outcomes for the three-player simple majority game, but is so in terms of payoffs. We conclude by generalizing the latter insight to the entire class of proper simple games.

Proper simple games are coalition function form games $(N, v)$ such that $v(N)=1$, for every $C \in \mathcal{N}$ it holds that $v(C) \in\{0,1\}$, and $v(C)=1$ implies $v(N \backslash C)=0$. Moreover, for $C, D \in \mathcal{N}$ such that $C \subseteq D, v(C)=1$ implies $v(D)=1$. The collection of winning coalitions of a proper simple game $(N, v)$ is denoted by $\mathcal{W}=\{C \in \mathcal{N} \mid v(C)=1\}$. A veto player is a player that belongs to every winning coalition. The set of veto players is given 
by $C^{*}=\cap_{C \in \mathcal{W}} C$. The coalition structure core of a proper simple game is non-empty if and only if there are veto players, so $C^{*} \neq \emptyset$.

Let $(N, v)$ be a proper simple game. We define the sets of outcomes $F$ and $\widetilde{F}$ by

$$
\begin{aligned}
& F= \begin{cases}\mathrm{CSC}, & \text { if } \mathrm{CSC} \neq \emptyset, \\
\{x \in X|\mathcal{P}(x) \cap(\mathcal{W} \backslash\{N\}) \neq \emptyset, \forall C \in \mathcal{P}(x) \backslash \mathcal{W},| C \mid=1\}, & \text { if } \mathrm{CSC}=\emptyset,\end{cases} \\
& \widetilde{F}= \begin{cases}\mathrm{CSC}, & \text { if } \mathrm{CSC} \neq \emptyset, \\
\left\{x \in X \mid \mathcal{P}(x) \cap \mathcal{W} \neq \emptyset, \exists i \in N \text { such that } u_{i}(x)=0\right\}, & \text { if } \operatorname{CSC}=\emptyset .\end{cases}
\end{aligned}
$$

It is easily verified that the definitions of $F$ and $\widetilde{F}$ are appropriate generalizations of the sets as defined in Example 3.6. In case CSC $=\emptyset$, the difference between $F$ and $\widetilde{F}$ is that outcomes where the grand coalition forms are included in the latter, as well as outcomes where non-winning coalitions need not be singletons. In payoff terms, there is no difference between $F$ and $\widetilde{F}$. For instance, the requirement for $\widetilde{F}$ that at least one player gets a payoff of zero in case CSC $=\emptyset$ is also satisfied for $F$, since if $x \in F$ then $\mathcal{P}(x)$ includes a winning coalition different from the grand coalition and the remaining coalitions in $\mathcal{P}(x)$ are required to be singletons. Any singleton coalition has a worth of zero, since otherwise the corresponding player would be a veto player, which is excluded when CSC $=\emptyset$.

The next result shows that for proper simple games the MDS and the MSS is equal to the set $F$ and the PEMDS to the set $\widetilde{F}$, from which payoff equivalence easily follows.

Theorem 5.5. Let $(N, v)$ be a proper simple game. The MDS and the MSS of $(N, v)$ are equal to $F$. The PEMDS of $(N, v)$ is equal to $\widetilde{F}$. The set of payoffs supported by the PEMDS, the MDS and the MSS coincide.

Proof. Assume first that CSC $\neq \emptyset$. By Theorems 3.3, 3.5, and 4.4, the MDS, PEMDS and MSS are all equal to CSC.

Assume next that $\mathrm{CSC} \neq \emptyset$. By Theorem 4.5 in Demuynck et al. (2019) it holds that the MSS is equal to the set $F$. It now follows from Theorem 5.4 that the MDS is equal to the set $F$.

We finally turn to the PEMDS. We show that the set $\widetilde{F}$ is a closed cycle, that is, for every $x \in \widetilde{F}$, it holds that $\widetilde{g}^{\mathbb{N}}(x)=\widetilde{F}$. We also argue that $\widetilde{F}$ satisfies Accessibility. It then follows easily that the PEMDS is equal to $\widetilde{F}$.

The proof of Theorem 4.5 in Demuynck et al. (2019) establishes that, for every $x \in F$, $f^{\infty}(x)=F$, and, for every $x \in X \backslash F, f^{\infty}(x) \cap F \neq \emptyset$. By Lemma 5.2 we then obtain that,

$$
\begin{aligned}
& \forall x \in F, g^{\mathbb{N}}(x)=F, \\
& \forall x \in X \backslash F, g^{\mathbb{N}}(x) \cap F \neq \emptyset .
\end{aligned}
$$

Since $F \subset \widetilde{F}$, it follows from (5.2) that $\widetilde{F}$ satisfies Accessibility. To prove that $\widetilde{F}$ is a closed cycle, we proceed in two steps. 
Step 1. For every $x \in \widetilde{F}, \widetilde{g}^{\mathbb{N}}(x) \supseteq \widetilde{F}$.

Let some $x, y \in \widetilde{F}$ be given. We distinguish four cases.

Case 1. $x, y \in F$.

It holds that $y \in g^{\mathbb{N}}(x) \subseteq \widetilde{g}^{\mathbb{N}}(x)$.

Case 2. $x \in F, y \in \widetilde{F} \backslash F$.

It holds that either $\mathcal{P}(y)=\{N\}$ or $\mathcal{P}(y)$ contains a winning coalition $C \subset N$ and a coalition with at least two players that is not winning. In the former case, let $i \in N$ be such that $u_{i}(y)=0$ and define the outcome $y^{\prime} \in X$ by $\mathcal{P}\left(y^{\prime}\right)=\{N \backslash\{i\},\{i\}\}$ and $u\left(y^{\prime}\right)=u(y)$. To demonstrate that $y^{\prime}$ is indeed an outcome, we show that $N \backslash\{i\}$ is a winning coalition. Suppose $N \backslash\{i\}$ is not a winning coalition. Then no subset of $N \backslash\{i\}$ is a winning coalition, so every winning coalition involves player $i$. But then $i$ is a veto player and the coalition structure core of $(N, v)$ is non-empty, a contradiction. Consequently, $N \backslash\{i\}$ is a winning coalition. In the latter case, define the outcome $y^{\prime} \in X$ by $\mathcal{P}\left(y^{\prime}\right)=\{C\} \cup\langle N \backslash C\rangle$ and $u\left(y^{\prime}\right)=u(y)$. Since the complement of a winning coalition is not winning, it is obvious that $y^{\prime}$ is an outcome. In both cases, it follows that $y^{\prime} \in[y]$, so $y \in \widetilde{g}\left(y^{\prime}\right)$. Since $x, y^{\prime} \in F$, we have by (5.1) that $y^{\prime} \in g^{\mathbb{N}}(x)$. We conclude that $y \in \widetilde{g}^{\mathbb{N}}(x)$.

Case 3. $x \in \widetilde{F} \backslash F, y \in F$.

By the same argument as in Case 2, there is $x^{\prime} \in F$ such that $x^{\prime} \in[x]$, so $x^{\prime} \in \widetilde{g}(x)$. Since $x^{\prime}, y \in F$, we have by (5.1) that $y \in g^{\mathbb{N}}(x)$. We conclude that $y \in \widetilde{g}^{\mathbb{N}}(x)$.

Case 4. $x \in \widetilde{F} \backslash F, y \in \widetilde{F} \backslash F$.

By the same argument as in Case 2, there is $x^{\prime} \in F$ such that $x^{\prime} \in[x]$ and there is $y^{\prime} \in F$ such that $y^{\prime} \in[y]$. Since $x^{\prime}, y^{\prime} \in F$, we have by (5.1) that $y^{\prime} \in g^{\mathbb{N}}\left(x^{\prime}\right)$. Since $x^{\prime} \in \widetilde{g}(x)$, $y^{\prime} \in g^{\mathbb{N}}\left(x^{\prime}\right)$ and $y \in \widetilde{g}\left(y^{\prime}\right)$, we conclude that $y \in \widetilde{g}^{\mathbb{N}}(x)$.

Step 2. For every $x \in \widetilde{F}, \widetilde{g}^{\mathbb{N}}(x) \subseteq \widetilde{F}$.

It is sufficient to show that, for every $x \in \widetilde{F}, \widetilde{g}(x) \subseteq \widetilde{F}$. Let some $x \in \widetilde{F}$ and some $y \in \widetilde{g}(x)$ be given. We distinguish four cases.

Case 1. $x \in F, y \in g(x)$.

It holds that $y \in g^{\mathbb{N}}(x)=F$, where the equality follows from (5.1).

Case 2. $x \in F, y \in[x]$.

Since $\mathcal{P}(x) \cap(\mathcal{W} \backslash N) \neq \emptyset$, there exists $i \in N$ such that $u_{i}(x)=0$ and $\mathcal{P}(y)$ must contain a winning coalition. It follows that $y \in \widetilde{F}$.

Case 3. $x \in \widetilde{F} \backslash F, y \in g(x)$.

It holds that either $\mathcal{P}(x)=\{N\}$ or $\mathcal{P}(x)$ contains a winning coalition $C \subset N$ and a coalition with at least two players that is not winning. In the former case, $y=x \in \widetilde{F} \backslash F$ or there is $D \in \mathcal{N}$ such that $y$ weakly dominates $x$ by $D$. The definition of weak dominance implies that $D$ is a proper subset of $N$, so $\mathcal{P}(y)=\{D\} \cup\langle N \backslash D\rangle$ and therefore $y \in F$. In the latter case $y=x \in \widetilde{F} \backslash F$ or there is $D \in \mathcal{W}$ such that $y$ weakly dominates $x$ by $D$, so 
$D \in \mathcal{P}(y) \cap \mathcal{W}$. By the definition of weak dominance it follows that $C \backslash D \neq \emptyset$, so $u_{i}(y)=0$ for every $i \in C \backslash D$. We have that $y \in \widetilde{F}$.

Case 4. $x \in \widetilde{F} \backslash F, y \in[x]$.

It clearly holds that $\mathcal{P}(y) \cap \mathcal{W} \neq \emptyset$ and there exists $i \in N$ such that $u_{i}(y)=0$, so $y \in \widetilde{F}$.

\section{Conclusion}

Since the coalition structure core of a coalition function form game may be empty, the literature has proposed non-empty generalizations like the payoff-equivalence minimal dominant set in Kóczy and Lauwers (2007) and the myopic stable set in Demuynck et al. (2019). An attractive feature of the myopic stable set is that it coincides with the coalition structure core whenever the coalition structure core is non-empty and provides a unique non-empty prediction otherwise. Kóczy and Lauwers (2007) make the same assertion for the payoff-equivalence minimal dominant set. Unfortunately, we identify some problems in the original definition of the payoff-equivalence minimal dominant set, correct them, and also suggest the minimal dominant set as an alternative. The minimal dominant set is shown to coincide with the coalition structure core whenever the coalition structure core is non-empty and provide a unique non-empty prediction otherwise. Both the myopic stable set and the minimal dominant set admit a dynamic interpretation in terms of coalitions that deviate with some probability whenever they can improve the utility of their members.

The minimal dominant set is a minimal set satisfying Closure and Sequential weak dominance. The myopic stable set is a minimal closed set satisfying Deterrence of external deviations and Asymptotic external stability. Contrary to the minimal dominant set, the myopic stable set requires every member of a deviating coalition to improve strictly. Otherwise, it can be shown that there is no substantial difference between Closure and Deterrence of external deviations. Sequential weak dominance requires that from any outcome outside the minimal dominant set it is possible to attain an outcome in the minimal dominant set by a finite number of weak dominations, whereas asymptotic external stability requires that from any outcome outside the myopic stable set it is possible to get arbitrary close to an outcome in the myopic stable set by a finite number of dominations. Finally, a myopic stable set is closed by definition, whereas no such requirement is made for the minimal dominant set.

The main result of the paper is that despite the subtle differences in the definition, the minimal dominant set is equivalent to the myopic stable set. We also argue that for important classes of coalition function form games, both concepts coincide with the payoffequivalence minimal dominant set. For instance, this is the case for generic coalition function form games, coalition function form games with a non-empty coalition structure 
core, and, when as far as payoffs are concerned, proper simple games.

\section{References}

Arnold, T. and U. Schwalbe, 2002, Dynamic coalition formation and the core, Journal of Economic Behavior \& Organization 49, 363-380.

Aumann, R. J. and J. H. Drèze, 1974, Cooperative games with coalition structures, International Journal of Game Theory 3, 217-237.

Béal, S., É. Rémila, and P. Solal, 2012, On the number of blocks required to access the core, Discrete Applied Mathematics 160, 925-932.

Béal, S., É. Rémila, and P. Solal, 2013a, Accessibility and stability of the coalition structure core, Mathematical Methods of Operations Research 78, 187-202.

Béal, S., É. Rémila, and P. Solal, 2013b, An optimal bound to access the core in TU-games, Games and Economic Behavior 80, 1-9.

Bhattacharya, A., and A. Ziad, 2006, The core as the set of eventually stable outcomes: A note, Games and Economic Behavior 54, 25-30.

Chen, B., S. Fujishige, and Z. Yang, 2016, Random decentralized market processes for stable job matchings with competitive salaries, Journal of Economic Theory 165, 25-36.

Demuynck, T., P. J. J. Herings, R. D. Saulle, and C. Seel, 2019, The myopic stable set for social environments, Econometrica 87, 111-138.

Diamantoudi, E., E. Miyagawa, and L. Xue, 2004, Random paths to stability in the roommate problem, Games and Economic Behavior 48, 18-28.

Green, J. R., 1974, The stability of Edgeworth's recontracting process, Econometrica 42, $21-34$.

Hart, S. and M. Kurz, 1983, Endogenous formation of coalitions, Econometrica 51, 1047 1064.

Herings, P. J. J., A. Mauleon, and V. Vannetelbosch, 2017, Stable sets in matching problems with coalitional sovereignty and path dominance, Journal of Mathematical Economics $71,14-19$.

Klaus, B. and F. Klijn, 2007, Paths to stability for matching markets with couples, Games and Economic Behavior 58, 154-171. 
Kóczy, L. Á., 2006, The core can be accessed with a bounded number of blocks, Journal of Mathematical Economics 43, 56-64.

Kóczy, L. Á., 2018, Partition function form games, Theory and Decision Library C 48, (Springer International Publishing), $312 \mathrm{p}$.

Kóczy, L. Á. and L. Lauwers, 2004, The coalition structure core is accessible, Games and Economic Behavior 48, 86-93.

Kóczy, L. Á. and L. Lauwers, 2007, The minimal dominant set is a non-empty coreextension, Games and Economic Behavior 61, 277-298.

Konishi, H. and D. Ray, 2003, Coalition formation as a dynamic process, Journal of Economic Theory 110, 1-41.

Neuefeind, W., 1974, A stochastic bargaining process for n-person games, Journal of Mathematical Economics 1, 175-191.

Packel, E. W., 1981, A stochastic solution concept for $n$-person games, Mathematics of Operations Research 6, 349-362.

Ray, D. and R. Vohra, 2014, Coalition Formation, in: H. P. Young and S. Zamir, eds., Handbook of Game Theory with Economic Applications 4, chap. 5 (North Holland), 239-326.

Ray, D. and R. Vohra, 2015, The Farsighted Stable Set, Econometrica 83, 977-1011.

Roth, A. E. and J. H. Vande Vate, 1990, Random paths to stability in two-sided matching, Econometrica 58, 1475-1480.

Sengupta, A. and K. Sengupta, 1994, Viable proposals, International Economic Review 35, $347-359$.

Sengupta, A. and K. Sengupta, 1996, A property of the core, Games and Economic Behavior 12, 266-273.

Stearns, R. E., 1968, Convergent transfer schemes for $n$-person games, Transactions of the American Mathematical Society 134, 449-459.

Wu, L. S. Y., 1977, A dynamic theory for the class of games with nonempty cores, SIAM Journal on Applied Mathematics 32, 328-338.

Yang, Y. Y., 2010, On the accessibility of the core, Games and Economic Behavior 69, 194 -199 . 
Yang, Y. Y., 2011, Accessible outcomes versus absorbing outcomes, Mathematical Social Sciences 62, 65-70.

Yang, Y .Y., 2020, On the characterizations of viable proposals, Working Paper, 1-25. 